\title{
Management and Treatment of Osteoporosis in Patients Receiving Long-term Glucocorticoid Treatment: Current Status of Adherence to Clinical Guidelines and Related Factors
}

\author{
Daijun Kirigaya $^{1,2}$, Takeo Nakayama ${ }^{2}$, Tatsuro Ishizaki ${ }^{2}$, Shunya Ikeda ${ }^{3}$ and Toshihiko Satoh ${ }^{4}$
}

\begin{abstract}
Objective The aim of this study was to evaluate the adherence of guidelines for the management and treatment of glucocorticoid-induced osteoporosis, and to investigate whether it is associated with factors such as age, gender, glucocorticoid dose, physician specialty, and size of facility.

Methods This was a cross-sectional study utilizing administrative data from a database of health insurance claims (2004-2007); 2,368 patients who received glucocorticoid treatment for $\geq 90$ days were extracted. The guideline adherence was determined by evaluations based on glucocorticoid prescription dose, prescription of anti-osteoporosis drugs, and whether or not bone mineral density was measured.

Results Overall proportion of guideline adherence was $23.3 \%$. In cases in which the equivalent dose of prednisolone was $<5 \mathrm{mg} / \mathrm{d}$ and $\geq 5 \mathrm{mg} / \mathrm{d}$, the adherence was $8.3 \%$ and $30.5 \%$ respectively. Factors correlating with low adherence included young age, male gender, and lower glucocorticoid doses. Surgery and otolaryngology specialties had lower adherence than internal medicine. Smaller clinical facilities had lower adherence than larger facilities.

Conclusion The adherence of guidelines for the management and treatment of glucocorticoid-induced osteoporosis is still low, and improvements in treatment quality can be expected through education of patient groups and medical care providers with large deviations from the guidelines.
\end{abstract}

Key words: clinical guidelines, glucocorticoid-induced osteoporosis, guideline adherence

(Intern Med 50: 2793-2800, 2011)

(DOI: 10.2169/internalmedicine.50.5266)

\section{Introduction}

Glucocorticoids are used widely for their strong antiinflammatory and immunosuppressive effects. There are many side effects to long-term glucocorticoid use, an important one of which is osteoporosis and subsequent bone fracture (1-3). A 1996 study by the American College of Rheumatology revealed that $20 \%$ of osteoporosis patients in the U.S. were induced by glucocorticoids and that $25 \%$ of patients under long-term glucocorticoid treatment had bone fractures (4). It has been reported that the risk of new verte- bral fractures reaches its peak at 3-6 months after initiation of oral glucocorticoid treatment (5), and the importance of early management has been recognized.

Since 1996, the efficacy of anti-osteoporosis drugs has been reported in some large-scale clinical trials (6). The efficacy of drugs used to treat glucocorticoid-induced osteoporosis (GIOP) has also been established. Specifically, bisphosphonates are effective in preventing and treating bone loss in GIOP patients (7-9). Although less effective, activated vitamin $\mathrm{D}_{3}$ and vitamin $\mathrm{K}_{2}$ also have been reported to prevent bone fractures $(10,11)$. In the U.S. and Europe, management guidelines for GIOP were published in

\footnotetext{
${ }^{1}$ Japan Association for Development of Community Medicine, Japan, ${ }^{2}$ Department of Health Informatics, Kyoto University School of Public Health, Japan, ${ }^{3}$ International University of Health and Welfare, Japan and ${ }^{4}$ Kitasato Clinical Research Center, Kitasato University School of Medicine, Japan

Received for publication February 3, 2011; Accepted for publication August 11, 2011

Correspondence to Dr. Takeo Nakayama, t-nakayama@umin.ac.jp
} 


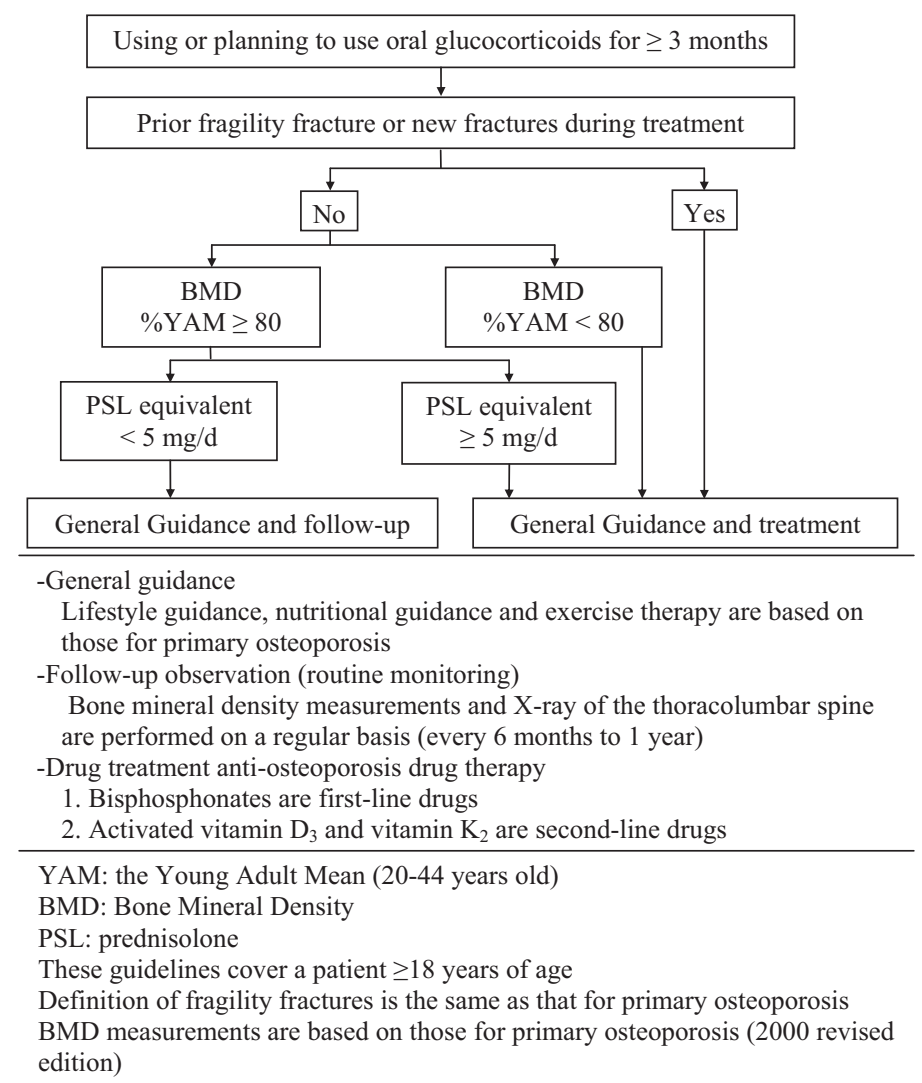

Figure 1. Guidelines on the management and treatment of glucocorticoid-induced osteoporosis in Japan (2004 edition) (13).

$1996(3,12)$ and have since been revised. In Japan, the "Guidelines for the Management and Treatment of Glucocorticoid-induced Osteoporosis" was released for the first time in 2004 (13). The guidelines apply to patients 18 years of age or older who are using or planning to use oral glucocorticoids for $\geq 3$ months, and they emphasize management and treatment from an early stage (Fig. 1).

After the release of the guidelines in Japan, no large-scale study on the state of adherence to guidelines in clinical practice has been conducted. Since glucocorticoids are used widely regardless of specialty, adherence to the guidelines may differ among specialties. In addition, osteoporosis is more frequently seen in postmenopausal women and the elderly (14), thus management and treatment may not be sufficiently conducted for males or young patients under longterm glucocorticoid treatment. In order to improve osteoporosis treatment, various trials have been conducted, some of which have been effective $(15,16)$. On the other hand, the previous trials on GIOP have shown low efficacies $(17,18)$, and it is important to know what characteristics of the patient and clinical setting should be intervened.

It has recently become clear that there is a discrepancy between clinical evidence and actual practice $(19,20)$. Improving the quality of clinical practice requires not only determining evidence, but also implementing the means to improve this evidence. The propagation of a recommended treatment through clinical guidelines is one method of disseminating evidence (21). Investigation of how well guide- lines are adhered to and the factors related to adherence will aid in recognizing the deviation of actual clinical practice from the guidelines. We used a database of health insurance claims (receipts) to study adherence to guidelines for the management and treatment of GIOP, and its relation to factors such as age, gender, glucocorticoid dose, physician specialty, and facility size.

\section{Materials and Methods}

\section{Study design}

The Japan Medical Data Center Co., Ltd (JMDC) (22) has constructed and run a database of health insurance claims data from health insurance unions in Japan: JMDCMDB (medical database). This database includes 530,000 cumulative insured persons, mainly company employees and their family members, from January 2004 to December 2007. Individuals who fulfilled the criteria for the guidelines were selected from the JMDC-MDB and a cross-sectional study was carried out.

\section{Participants}

This study utilized cases in which oral glucocorticoids were prescribed continuously for $\geq 90$ days. We extracted cases from the JMDC-MDB that included at least 3 months of treatment in which oral glucocorticoids were prescribed for at least 28 days during the study period and in which the 
- Patients taking $<5 \mathrm{mg} / \mathrm{d}$ PSL equivalent

A) Prescription of anti-osteoporosis drugs (drug therapy) (Drug therapy was assumed to be initiated due to a history of fragility fracture or BMD value)

B) Routine measurement of BMD (monitoring)

- Patients taking $\geq 5 \mathrm{mg} / \mathrm{d}$ PSL equivalent

C) Prescription of anti-osteoporosis drugs (drug therapy)

Figure 2. Standards for determining adherence to the guidelines.

patient was 18 years of age or older. Of these cases, exclusion criteria were those in which the prescription was continuous for $<90$ days; hypopituitarism or adrenal gland dysfunction necessitated supplementation of adrenocortical hormones; special conditions such as palliative care were thought to be due to malignant tumors and the prescribed dose of glucocorticoids was unclear.

The background information obtained from the JMDCMDB for each participant included year of birth, gender, details of oral glucocorticoid prescriptions, physician specialty, and facility size. The prescribed dose of glucocorticoids was calculated by converting the dose to the equivalent of prednisolone (PSL). When the dose changed during the course of the treatment, the maximum mean dose of 90 continuous days was used. When oral glucocorticoids were prescribed by physicians in multiple specialties, the specialty which was primarily responsible for prescribing glucocorticoids was used. Facility size was divided into four groups: clinic (0-19 beds), small hospital (20-199 beds), medium-sized hospital (200-499 beds), and large hospital ( $\geq 500$ beds).

\section{Measurement of adherence to guidelines}

Adherence to guidelines was used as the main outcome measurement. According to the flowchart in the guidelines (Fig. 1), anti-osteoporosis drug therapy is recommended for patients with previous fragility fractures, patients who had a bone fracture occur during treatment, and those in whom bone mineral density (BMD) was $<80 \%$ of the young adult mean (YAM). The guidelines list bisphosphonates as the drugs of choice for this treatment, with activated vitamin $\mathrm{D}_{3}$ or vitamin $K_{2}$ as alternatives. Since the disease name on the health insurance claims did not always represent the actual disease and they lacked information on results of medical tests performed, adherence to guidelines in this study was evaluated based on glucocorticoid prescription dose, prescription of anti-osteoporosis drugs, and whether or not BMD was measured (Fig. 2).

Bone fracture risk increases dose-dependently with glucocorticoids, and it has been reported that $5 \mathrm{mg} / \mathrm{d}$ PSL is the threshold dose beyond which bone fracture risk increases (5). Consequently, the Japanese guidelines recommend routine monitoring (every 6 months to 1 year) by BMD measurement and X-ray of the thoracolumbar spine for patients taking $<5 \mathrm{mg} / \mathrm{d}$ PSL, and drug therapy regardless of BMD value if the PSL dose is $\geq 5 \mathrm{mg} / \mathrm{d}$. In cases in which an anti-osteoporosis drug was prescribed during glucocorticoid treatment with $<5 \mathrm{mg} / \mathrm{d}$ PSL, it was assumed that prescription of anti-osteoporosis drugs was initiated due to a prior fragility fracture or low BMD measurements, and it was considered as adherence to guidelines (Fig. 2, A). When anti-osteoporosis drugs were not prescribed, routine monitoring of BMD as adherence to guidelines was considered (Fig. 2, B). In cases in which the PSL dose was $\geq 5 \mathrm{mg} / \mathrm{d}$, prescription of an anti-osteoporosis drug during glucocorticoid treatment was considered as adherence to guidelines (Fig. 2, C).

\section{Statistical analysis}

First, the number of participants in this study was determined and characteristics of the patient (gender, age, and glucocorticoid dose) and of the clinical setting (physician specialty and facility size) were noted. Male and female patient attributes were compared by using the chi-square test and t-test. Adherence to the guidelines was determined based on glucocorticoid dose, prescription of antiosteoporosis drugs, and BMD measurement. A univariate analysis was performed to determine the correlation between adherence to the guidelines and five factors (gender, age, glucocorticoid dose, physician specialty, and facility size). Next, multiple logistic regression analyses were performed using adherence to the guidelines as the dependent variable and each factor as the independent variable. Furthermore, cases were divided into two groups based on PSL dose $(\geq 5$ $\mathrm{mg} / \mathrm{d}$ or $<5 \mathrm{mg} / \mathrm{d}$ ) since guideline adherence standards are different for these two groups were divided, and a stratified analysis was performed. Stratified analyses by gender, age and facility size were also performed. Differences were considered to be statistically significant at $\mathrm{p}<0.05$ for all statistical tests. All analyses were performed using STATA version 11 (Stata Corp., College Station, TX, USA).

This study was approved by the Ethics Committee, Kyoto University Graduate School of Medicine.

\section{Results}

Initial search was matched criteria of a patient $\geq 18$ years of age for whom oral glucocorticoids were prescribed for $\geq$ 28 days/month for $\geq 3$ months during the study period, and 


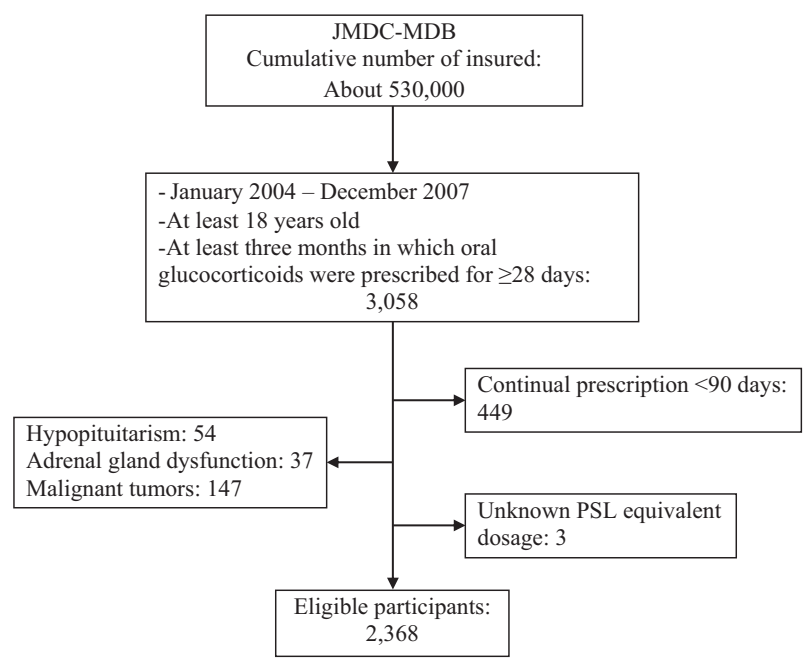

Figure 3. Flowchart for the selection of study participants.

3,058 cases were found. Among these cases, 449 patients who were not prescribed continually for $\geq 90$ days, 54 patients with hypopituitarism, 37 with adrenal gland dysfunction, 147 with suspected malignant tumors, and 3 with unknown glucocorticoid dose were excluded. The remaining 2,368 patients were included as the present participants (Fig. 3).

\section{Characteristics of participants}

Table 1 summarizes the characteristics of the 2,368 eligible participants. Mean age was 45.9 years (range: 19-98 years), and $53.5 \%$ of participants were female. Mean dose of glucocorticoids was $6.6 \mathrm{mg} / \mathrm{d}$ PSL equivalent, and the dose was $<5 \mathrm{mg} / \mathrm{d}$ for approximately one-third of the cases. The majority of physicians prescribing glucocorticoids were in the internal medicine specialty group (57.4\%). Approximately half of the facilities were clinics (0-19 beds). In $22.9 \%$ of the cases, the first or second choice osteoporosis medication was prescribed during glucocorticoid treatment. In some cases, multiple osteoporosis drugs were prescribed, but activated vitamin $D_{3}$ was the predominantly prescribed drug and vitamin $\mathrm{K}_{2}$ was seldom prescribed. BMD was measured in only $4.7 \%$ of patients not receiving osteoporosis treatment, revealing its infrequent use as a monitoring method. Gender comparison revealed that males were significantly younger than female participants $(\mathrm{p}<0.001)$. The majority of participants that received glucocorticoid prescriptions from internal medicine and orthopedic specialists were female, while those participants who were prescribed glucocorticoids by physicians in dermatology and otolaryngology were predominantly male. More males were treated in clinics and more females were treated in large hospitals. Males were significantly less likely to receive osteoporosis treatment and BMD measurement.

Table 2 shows the treated underlying diseases according to physician specialty. The main three diseases, allergic rhinitis, rheumatoid arthritis and atopic dermatitis, constituted up to half of the overall underlying diseases. The most common disease was rheumatoid arthritis for orthopedic surgery, and allergic rhinitis for otolaryngology. In the other physician specialties, there were various underlying diseases.

\section{Main analysis}

Adhering to the guidelines was a total of 551 cases (23.3\%). Amongst the patients taking an equivalent of $<5$ $\mathrm{mg} / \mathrm{d}$ PSL, 64 cases $(8.3 \%)$ were adhering to the guidelines (Table 3). These cases included 56 patients (7.3\%) who received osteoporosis medication during glucocorticoid treatment and 8 patients $(1.0 \%)$ who were not prescribed osteoporosis medication, but had routine BMD measurements (every 6 months to 1 year). Among these patients taking $\geq 5$ $\mathrm{mg} / \mathrm{d}$ PSL, 487 cases (30.5\%) in which osteoporosis medications were prescribed during glucocorticoid treatment were classified as adhering to the guidelines.

The adherence for each factor and odds ratios (ORs) before and after adjustment by logistic regression was calculated (Table 4). The adjusted odds ratio (AOR) was higher in females and increased with age. Glucocorticoid dose was divided into groups of $5 \mathrm{mg}$ increments and each group was evaluated separately. ORs decreased after adjustments, but increased dose-dependently. Differences among specialties were observed, and ORs were smaller for surgery and otolaryngology compared to internal medicine. ORs were larger for larger facilities, despite the decrease after adjustment. Due to a small amount of missing data, a sensitivity analysis was not performed.

\section{Subgroup analysis}

Stratified analysis was performed by dividing patients into those taking $\geq 5 \mathrm{mg} / \mathrm{d}$ PSL and those taking $<5 \mathrm{mg} / \mathrm{d}$ PSL, and calculating AOR for each group (Table 5). In cases with PSL dose $<5 \mathrm{mg} / \mathrm{d}$, OR was greater for females, physicians in the orthopedic specialty, and small-scale hospitals, while ORs tended to be smaller for otolaryngology and dermatology specialists. In cases with PSL dose $\geq 5 \mathrm{mg} / \mathrm{d}$, results were similar, except for the lack of a significant difference in otolaryngology.

General knowledge that osteoporosis frequently occurs after menopause may increase clinicians' attitude for providing examination and treatment. Therefore, stratified analysis was performed based on gender and the possibility of menopause, with a cut-off age of 50 years old (Table 6). For male, ORs tended to be much larger as the prescribed dose of glucocorticoids increased regardless of the age compared with females. For females, the influence of facility size was less in the older group than in the younger one.

Due to the lack of examination resources, measuring BMD is difficult to do at clinics relative to medium-sized or large hospitals. Stratified analysis based on facility size showed that female or older patients were more likely to be treated according to guidelines' recommendation at clinics (Table 7). 
Table 1. Characteristics of Participants

\begin{tabular}{|c|c|c|c|}
\hline & $\begin{array}{c}\text { Total } \\
\mathrm{n}=2,368\end{array}$ & $\begin{array}{c}\text { Males } \\
\mathrm{n}=1,100\end{array}$ & $\begin{array}{c}\text { Females } \\
\mathrm{n}=1,268\end{array}$ \\
\hline Mean age, year \pm SD & $45.9 \pm 13.4$ & $44.4 \pm 12.9$ & $47.3 \pm 13.8$ \\
\hline Gender, male (\%) & $1100(46.5)$ & & \\
\hline \multicolumn{4}{|l|}{ Glucocorticoid dose (\%) } \\
\hline $\mathrm{PSL}<5 \mathrm{mg} / \mathrm{d}$ & $772(32.6)$ & $364(33.1)$ & $408(32.2)$ \\
\hline $\mathrm{PSL} \geq 5 \mathrm{mg} / \mathrm{d}$ & $1596(67.4)$ & $736(66.9)$ & $860(67.8)$ \\
\hline \multicolumn{4}{|l|}{ Physician specialty (\%) } \\
\hline Internal medicine & $1359(57.4)$ & $577(52.5)$ & $782(61.7)$ \\
\hline Surgery & $176(7.4)$ & $92(8.4)$ & $84(6.6)$ \\
\hline Orthopedic surgery & $237(10.0)$ & $72(6.5)$ & $165(13.0)$ \\
\hline Dermatology & $365(15.4)$ & $107(9.7)$ & $86(6.8)$ \\
\hline Otolaryngology & $193(8.2)$ & $234(21.3)$ & $131(10.3)$ \\
\hline Other specialties & $38(1.6)$ & $18(1.6)$ & $20(1.6)$ \\
\hline \multicolumn{4}{|l|}{ Facility size (\%) } \\
\hline Clinic & $1204(50.8)$ & $598(54.4)$ & $606(47.8)$ \\
\hline Small hospital & $145(6.1)$ & $69(6.3)$ & $76(6.0)$ \\
\hline Medium-sized hospital & $265(11.2)$ & $112(10.2)$ & $153(12.1)$ \\
\hline Large hospital & $754(31.8)$ & $321(29.2)$ & $433(34.1)$ \\
\hline \multicolumn{4}{|l|}{ Anti-osteoporosis drug (\%) } \\
\hline Bisphosphonates & $286(12.1)$ & $57(5.2)$ & $229(18.1)$ \\
\hline Activated vitamin $\mathrm{D}_{3}$ & $337(14.2)$ & $99(9.0)$ & $238(18.8)$ \\
\hline Vitamin $\mathrm{K}_{2}$ & $49(2.1)$ & $16(1.5)$ & $33(2.6)$ \\
\hline Other & $101(4.3)$ & $16(1.5)$ & $85(6.7)$ \\
\hline First or second choice treatment & $543(22.9)$ & $144(13.1)$ & $399(31.5)$ \\
\hline \multicolumn{4}{|l|}{ BMD measurement (\%) } \\
\hline During osteoporosis treatment & $194 / 543(35.7)$ & $33 / 144(22.9)$ & $151 / 399(37.8)$ \\
\hline Before osteoporosis treatment & $86 / 1825(4.7)$ & $21 / 956(2.2)$ & $65 / 869(7.5)$ \\
\hline
\end{tabular}

Facility size: clinic (0-19 beds), small hospital (20-199 beds), medium-sized hospital (200-499 beds), and large hospital ( $\geq 500$ beds)

Table 2. Treated Underlying Disease of Each Physician Specialty (The Main Three Diseases)

\begin{tabular}{llll}
\hline Physician specialty (N) & \multicolumn{3}{c}{ The main three diseases (N) } \\
\hline Overall (2368) & Allergic rhinitis (463) & Rheumatoid arthritis (407) & Atopic dermatitis (183) \\
Internal medicine (1359) & Allergic rhinitis (218) & Rheumatoid arthritis (209) & SLE (144) \\
Surgery (176) & Allergic rhinitis (31) & After the organ plant (25) & Atopic dermatitis (16) \\
Orthopedic surgery (237) & Rheumatoid arthritis (188) & Allergic rhinitis (10) & Bronchial asthma (5) \\
Otolaryngology (193) & Allergic rhinitis (162) & Bronchial asthma (14) & Sudden deafness (4) \\
Dermatology (365) & Atopic dermatitis (125) & Urticaria (52) & Allergic rhinitis (24) \\
\hline
\end{tabular}

Table 3. Guideline Adherence

\begin{tabular}{cc}
\hline & Guideline adherence \\
& $\mathrm{N}(\%)$ \\
\hline Total & $551 / 2368(23.3)$ \\
PSL $<\mathbf{5 m g / d}$ & $64 / 772(8.3)$ \\
PSL $\geq \mathbf{5 m g / d}$ & $487 / 1596(30.5)$ \\
\hline
\end{tabular}

\section{Discussion}

The overall proportion of adherence of $23.3 \%$ in this study was low compared to a U.S. study, in which the recommended acute, chronic, and preventative treatment was performed in about $50 \%$ of cases (19). The adherence was particularly low for cases with PSL dose $<5 \mathrm{mg} / \mathrm{d}$ and rou- tine monitoring by BMD measurement was rarely performed. When prescribing glucocorticoids for a long period, even in low dosages, physicians should take measures to monitor for potential osteoporosis. Proportion of adherence to the guidelines was $30.5 \%$ for cases in which antiosteoporosis drug therapy is recommended (PSL dose $\geq 5$ $\mathrm{mg} / \mathrm{d})$. In the U.S., where guidelines were implemented in 1996, the frequency of BMD measurement among patients receiving glucocorticoids in 2001-2003 increased to about triple compared with 1995-1998, and prescription of bisphosphonates increased to double or triple (23). In other countries as well, treatment of GIOP has improved in recent years (24). The proportion of patients receiving drug therapy is still low in Japan compared to the U.S., but an increase can be expected if the guidelines are propagated.

Factors associated with a high adherence in this study in- 
Table 4. Odds Ratio (before and after Adjustment) for Each Factor

\begin{tabular}{|c|c|c|c|}
\hline & $\begin{array}{c}\text { Guideline adherence } \\
\mathrm{N}(\%)\end{array}$ & $\begin{array}{c}\text { Odds ratio } \\
(95 \% \mathrm{CI})\end{array}$ & $\begin{array}{c}\text { Adjusted odds ratio } \\
(95 \% \mathrm{CI})\end{array}$ \\
\hline \multicolumn{4}{|l|}{ Age } \\
\hline 10 year increase & & $1.25(1.17-1.34)$ & $1.36(1.25-1.48)$ \\
\hline \multicolumn{4}{|l|}{ Gender } \\
\hline Male & $145 / 1100(13.2)$ & 1 & 1 \\
\hline Female & $406 / 1268(32.0)$ & $3.10(2.51-3.85)$ & $3.46(2.70-4.43)$ \\
\hline \multicolumn{4}{|l|}{ Glucocorticoid dose } \\
\hline$<5 \mathrm{mg} / \mathrm{d}$ & $64 / 772(8.3)$ & 1 & 1 \\
\hline$\geq 5,<10 \mathrm{mg} / \mathrm{d}$ & $217 / 1014$ (21.4) & $3.01(2.23-4.11)$ & $2.10(1.52-2.91)$ \\
\hline$\geq 10,<15 \mathrm{mg} / \mathrm{d}$ & $149 / 368(40.5)$ & $7.53(5.35-10.63)$ & $5.49(3.76-8.03)$ \\
\hline$\geq 15 \mathrm{mg} / \mathrm{d}$ & $121 / 214(56.5)$ & $14.40(9.76-21.25)$ & $10.15(6.55-15.73)$ \\
\hline \multicolumn{4}{|l|}{ Physician specialty } \\
\hline Internal medicine & $414 / 1359(30.5)$ & 1 & 1 \\
\hline Surgery & $17 / 176(9.7)$ & $0.24(0.14-0.41)$ & $0.28(0.16-0.48)$ \\
\hline Orthopedic surgery & $61 / 237(25.7)$ & $0.79(0.57-1.09)$ & $1.30(0.91-1.88)$ \\
\hline Otolaryngology & $5 / 193(2.6)$ & $0.06(0.02-0.14)$ & $0.27(0.11-0.69)$ \\
\hline Dermatology & $50 / 365(13.7)$ & $0.36(0.26-0.50)$ & $0.81(0.56-1.18)$ \\
\hline \multicolumn{4}{|l|}{ Facility size } \\
\hline Clinic & $99 / 1204(8.2)$ & 1 & 1 \\
\hline Small hospital & $41 / 145(28.3)$ & $4.40(2.83-6.77)$ & $2.77(1.75-4.38)$ \\
\hline Medium-sized hospital & $101 / 265(38.1)$ & $6.87(4.92-9.60)$ & $3.86(2.70-5.53)$ \\
\hline Large hospital & $310 / 754(41.1)$ & $7.79(6.03-10.12)$ & $4.88(3.67-6.50)$ \\
\hline
\end{tabular}

Table 5. Stratified Analysis Based on Glucocorticoid Dose (Adjusted Odds Ratio)

\begin{tabular}{|c|c|c|}
\hline & $\begin{array}{c}\mathrm{PSL}<5 \mathrm{mg} / \mathrm{d} \\
(95 \% \mathrm{CI}) \\
\mathrm{n}=772\end{array}$ & $\begin{array}{c}\mathrm{PSL} \geq 5 \mathrm{mg} / \mathrm{d} \\
(95 \% \mathrm{CI}) \\
\mathrm{n}=1,596\end{array}$ \\
\hline \multicolumn{3}{|l|}{ Age } \\
\hline 10 year increase & $1.37(1.11-1.70)$ & $1.36(1.24-1.49)$ \\
\hline \multicolumn{3}{|l|}{ Gender } \\
\hline Male & 1 & 1 \\
\hline Female & $8.37(3.45-20.34)$ & $3.15(2.42-4.09)$ \\
\hline \multicolumn{3}{|l|}{ Physician specialty } \\
\hline Internal medicine & 1 & 1 \\
\hline Surgery & $0.31(0.04-2.40)$ & $0.28(0.16-0.49)$ \\
\hline Orthopedic surgery & $2.01(1.01-4.01)$ & $1.11(0.72-1.72)$ \\
\hline Otolaryngology & $0.13(0.02-0.98)$ & $0.41(0.14-1.22)$ \\
\hline Dermatology & $0.53(0.20-1.44)$ & $0.91(0.60-1.38)$ \\
\hline \multicolumn{3}{|l|}{ Facility size } \\
\hline Clinic & 1 & 1 \\
\hline Small hospital & $5.29(1.97-14.19)$ & $2.38(1.42-4.00)$ \\
\hline Medium-sized hospital & $2.39(0.92-6.21)$ & $4.07(2.74-6.06)$ \\
\hline Large hospital & $4.24(2.17-8.28)$ & $4.93(3.57-6.80)$ \\
\hline
\end{tabular}

cluded old age, female gender, and larger doses of glucocorticoids. In particular, the implementation of guidelines' recommendation was sharply improved as prescribed dose of glucocorticoids increased in male. The influence of facility size was less in the older female group than in the younger group. It may be because clinicians at the clinic know the number of patients with menopausal osteoporosis increases with aging and they tended to implement guidelines' recommendation for older females even at clinics. On the other hand, attention towards the guidelines for young patients and males who receive long-term glucocorticoid treatment should be promoted to minimize the lack of appropriate treatment for these patients. In terms of physician specialty, the adherence was lower in surgery and otolaryngology compared to internal medicine. These specialties see fewer cases of osteoporosis, thus emphasis should be made to increase guideline awareness. Smaller facilities also showed lower adherence. Factors such as difficulty of immediate access to other specialties or clinical tests like BMD measurement may be possible reasons. Stratified analysis based on facility size showed that female or older ones were more likely to be treated according to guidelines' recommendation at clinics. Similar findings were obtained when stratified analyses were performed considering menopause; the cut-off age was 50 years old in the present study. Stratified analysis based on glucocorticoid dose showed some differences between PSL dose $\geq 5 \mathrm{mg} / \mathrm{d}$ and $<5 \mathrm{mg} / \mathrm{d}$. In the PSL dose $<5 \mathrm{mg} / \mathrm{d}$, trends of these cases were likely similar to those of cases of anti-osteoporosis treatment in the entire population since PSL equivalent glucocorticoid doses were low.

Although the receipt database is not research-oriented, such a database can be useful for research if its limitations (for example, the underlying disease name is unclear, and it is not understood whether it is facilities where BMD measurement is possible) are well understood. JMDC-MDB comprises data only from several health insurance unions, and its patient population is younger than the general patient population in Japan and sampling bias of participants is possible. Moreover, the present study is a simple cross-sectional study examining four years as a whole, so the differences of period of participants' adherence to guidelines were not considered. Many clinical guidelines have been instituted in the past decade in Japan $(25,26)$. However, there have been few reports on how well these guidelines have been followed. By using the receipt database, investigations of actual treatments in relation to other clinical guidelines, and long-term 
Table 6. Stratified Analysis by Gender and Age Group (Adjusted Odds Ratio)

\begin{tabular}{|c|c|c|c|c|}
\hline & $\begin{array}{c}\text { Male, Age }<50 \\
(95 \% \text { CI }) \\
n=693\end{array}$ & $\begin{array}{c}\text { Male, Age } \geq 50 \\
(95 \% \text { CI }) \\
n=407\end{array}$ & $\begin{array}{c}\text { Female, Age }<50 \\
(95 \% \mathrm{CI}) \\
\mathrm{n}=721\end{array}$ & $\begin{array}{c}\text { Female, Age } \geq 50 \\
(95 \% \mathrm{CI}) \\
n=547\end{array}$ \\
\hline \multicolumn{5}{|l|}{ Glucocorticoid } \\
\hline$<5 \mathrm{mg} / \mathrm{d}$ & 1 & 1 & 1 & 1 \\
\hline$\geq 5,<10 \mathrm{mg} / \mathrm{d}$ & $6.70(1.51-29.79)$ & $4.06(1.21-10.98)$ & $1.66(0.93-2.96)$ & $1.67(1.05-2.66)$ \\
\hline$\geq 10,<15 \mathrm{mg} / \mathrm{d}$ & $13.28(2.92-60.52)$ & $6.39(1.72-19.32)$ & $4.45(2.33-8.54)$ & $5.89(3.10-11.22)$ \\
\hline$\geq 15 \mathrm{mg} / \mathrm{d}$ & $34.17(7.28-160.4)$ & $10.56(2.94-38.02)$ & $8.52(4.16-17.46)$ & $4.94(1.87-13.12)$ \\
\hline \multicolumn{5}{|l|}{ Physician specialty } \\
\hline Internal medicine & 1 & 1 & 1 & 1 \\
\hline Surgery & $0.20(0.05-0.91)$ & $0.60(0.21-1.77)$ & $0.27(0.11-0.69)$ & $0.17(0.06-0.51)$ \\
\hline Orthopedic surgery & $1.16(0.13-10.59)$ & $0.73(0.23-2.36)$ & $1.82(0.95-3.48)$ & $1.16(0.71-1.92)$ \\
\hline Otolaryngology & $0.92(0.19-4.49)$ & $0.67(0.08-5.66)$ & $0.28(0.06-1.36)$ & $0.13(0.02-0.97)$ \\
\hline Dermatology & $1.84(0.89-2.29)$ & $0.63(0.24-1.70)$ & $0.76(0.39-1.52)$ & $0.49(0.22-1.09)$ \\
\hline \multicolumn{5}{|l|}{ Facility size } \\
\hline Clinic & 1 & 1 & 1 & 1 \\
\hline Small hospital & $4.37(1.11-17.30)$ & $1.97(0.53-7.41)$ & $5.08(1.98-13.05)$ & $2.52(1.27-4.99)$ \\
\hline Medium-sized & $9.40(3.44-25.74)$ & $4.92(1.79-13.54)$ & $8.13(4.16-15.89)$ & $1.59(0.90-2.83)$ \\
\hline Large hospital & $10.12(4.38-23.39)$ & $7.17(3.05-16.91)$ & $7.07(4.10-12.20)$ & $2.66(1.68-4.22)$ \\
\hline
\end{tabular}

Table 7. Stratified Analysis Based on Facility Size (Adjusted Odds Ratio)

\begin{tabular}{lcc}
\hline & $\begin{array}{c}\text { Clinic } \\
(95 \% \mathrm{CI}) \\
\mathrm{n}=1204\end{array}$ & $\begin{array}{c}\text { Medium-sized and } \\
\text { Large hospital } \\
(95 \% \mathrm{CI}) \\
\mathrm{n}=1019\end{array}$ \\
\hline $\begin{array}{l}\text { Age } \\
10 \text { year increase }\end{array}$ & $1.87(1.56-2.25)$ & $1.19(1.07-1.31)$ \\
Gender & & 1 \\
$\quad$ Male & 1 & 1 \\
Female & $5.42(2.95-9.96)$ & $2.77(2.07-3.70)$ \\
Glucocorticoid dose & & 1 \\
$<5$ mg/d & $1.70(0.99-2.92)$ & $2.40(1.51-3.82)$ \\
$\geq 5,<10$ mg/d & $6.85(3.26-14.42)$ & $5.45(3.27-9.09)$ \\
$\geq 10,<15$ mg/d & $32.74(9.98-107.3)$ & $9.55(5.52-16.52)$ \\
$\geq 15$ mg/d & & 1 \\
Physician specialty & $1.11(0.71-1.75)$ \\
Internal medicine & $0.28(0.08-0.99)$ & $0.29(0.16-0.53)$ \\
Surgery & $2.05(1.19-3.56)$ & $0.95(0.57-1.59)$ \\
Orthopedic surgery & $0.23(0.06-0.99)$ & $0.42(0.12-1.56)$ \\
Otolaryngology & $0.52(0.23-1.19)$ & \\
Dermatology & &
\end{tabular}

changes in treatment may be possible.

Although the guidelines list the first and second choice treatments for drug therapy, the efficacy of two groups is very different $(27,28)$. After the issuance of the guideline in Japan, there came some promising drugs. Teriparatide, a recombinant human parathyroid hormone, is more effective in increasing BMD than alendronate, a bisphosphonate, and the prescription for high risk patients (postmenopausal women and men age $\geq 50$ years) is recommended by American College of Rheumatology $2010(29,30)$. The present study investigated the adherence to the current Japanese guidelines published in 2004, but guidelines will be revised considering new evidence in the near future.
The present findings revealed that the current state of the management of osteoporosis in patients receiving chronic glucocorticoid therapy in Japan was found to be unsatisfactory in terms of established evidence. Potential factors for low adherence included young age, male gender, lower glucocorticoid dose, prescription by surgery and otolaryngology specialists, and smaller clinical facilities. The most common barriers in the adherence to the guideline are the provider factor, the patient factor, and the system factor (31). For example, poor knowledge is included in provider barriers, nonadherence is included in patient barriers, and obtaining DXA and computerized ordering system are included in system barriers. Provider education, patient educational handouts and computerized clinical reminders are suggested for guideline adherence improvement. Improvements in treatment quality can be expected by educating particular characteristic factors of the patients and the clinical settings associated with large deviations from guideline recommendations.

The authors state that they have no Conflict of Interest (COI).

\section{Acknowledgement}

We thank the JMDC for providing the database freely since this study was conducted as part of the activities of the research group for health administrative data. This study is partly supported by a grant-in-aid by the Ministry of Health, Labour and Welfare, Japan.

Grants:

This study is partly supported by the grant-in-aid by the Ministry of Health, Labour and Welfare, Japan.

\section{References}

1. Reid IR, Heap SW. Determinants of vertebral mineral density in patients receiving long-term glucocorticoid therapy. Arch Intern 
Med 150: 2545-2548, 1990.

2. Saag KG, Koehnke R, Caldwell JR, et al. Low dose long-term corticosteroid therapy in rheumatoid arthritis: an analysis of serious adverse events. Am J Med 96: 115-123, 1994.

3. Recommendations for the prevention and treatment of glucocorticoid-induced osteoporosis. American College of Rheumatology Task Force on Osteoporosis Guidelines. Arthritis Rheum 39: 1791-1801, 1996.

4. van Staa TP, Leufkens HG, Cooper C. The epidemiology of corticosteroid-induced osteoporosis: a meta-analysis. Osteoporos Int 13: 777-787, 2002.

5. Steinbuch M, Youket TE, Cohen S. Oral glucocorticoid use is associated with an increased risk of fracture. Osteoporos Int 15: 323-328, 2004.

6. Black DM, Cummings SR, Karpf DB, et al. Randomised trial of effect of alendronate on risk of fracture in women with existing vertebral fractures. Fracture Intervention Trial Research Group. Lancet 348: 1535-1541, 1996.

7. Saag KG, Emkey R, Schnitzer TJ, et al. Alendronate for the prevention and treatment of glucocorticoid-induced osteoporosis. Glucocorticoid-Induced Osteoporosis Intervention Study Group. N Engl J Med 339: 292-299, 1998.

8. Homik J, Cranney A, Shea B, et al. Bisphosphonates for steroid induced osteoporosis. Cochrane Database Syst Rev 2: CD001347, 2000.

9. Mok CC, Tong KH, To CH, Siu YP, Ma KM. Risedronate for prevention of bone mineral density loss in patients receiving highdose glucocorticoids: a randomized double-blind placebocontrolled trial. Osteoporos Int 19: 357-364, 2008.

10. de Nijs RN, Jacobs JW, Algra A, Lems WF, Bijlsma JW. Prevention and treatment of glucocorticoid-induced osteoporosis with active vitamin D3 analogues: a review with meta-analysis of randomized controlled trials including organ transplantation studies. Osteoporos Int 15: 589-602, 2004.

11. Tanaka I, Oshima H. A longitudinal study of diagnosis and treatment for glucocorticoid-induced osteoporosis. Osteoporosis Jpn 11: 11-14, 2003 (in Japanese).

12. Eastell R, Reid DM, Compston J, et al. A UK Consensus Group on management of glucocorticoid-induced osteoporosis: an update. J Intern Med 244: 271-292, 1998.

13. Nawata $H$, Soen $S$, Takayanagi $R$, et al. Guidelines on the management and treatment of glucocorticoid-induced osteoporosis of the Japanese Society for Bone and Mineral Research (2004). J Bone Miner Metab 23: 105-109, 2005.

14. Ross PD, Davis JW, Epstein RS, Wasnich RD. Pre-existing fractures and bone mass predict vertebral fracture incidence in women. Ann Intern Med 114: 919-923, 1991.

15. Feldstein A, Elmer PJ, Smith DH, et al. Electronic medical record reminder improves osteoporosis management after a fracture: a randomized, controlled trial. J Am Geriatr Soc 54: 450-457, 2006.

16. Polinski JM, Patrick A, Truppo $C$, et al. Interactive voice response telephone calls to enhance bone mineral density testing. Am J Manag Care 12: 321-325, 2006.

17. Curtis JR, Westfall AO, Allison J, et al. Challenges in improving the quality of osteoporosis care for long-term glucocorticoid users: a prospective randomized trial. Arch Intern Med 167: 591-596, 2007.

18. Chitre MM, Hayes W. 3-year results of a member and physician intervention to reduce risk associated with glucocorticoid-induced osteoporosis in a health plan. J Manag Care Pharm 14: 281-290, 2008.

19. McGlynn EA, Asch SM, Adams J, et al. The quality of health care delivered to adults in the United States. N Engl J Med 348: 2635-2645, 2003.

20. Wenger NS, Solomon DH, Roth CP, et al. The quality of medical care provided to vulnerable community-dwelling older patients. Ann Intern Med 139: 740-747, 2003.

21. Nakayama T. Evidence-based healthcare and health informatics: derivations and extension of epidemiology. J Epidemiol 16: 93100, 2006.

22. Kimura $S$, Sato $T$, Ikeda $S$, Noda $M$, Nakayama T. Development of a database of health insurance claims: standardization of disease classifications and anonymous record linkage. J Epidemiol 20: 413-419, 2010.

23. Curtis JR, Westfall AO, Allison JJ, et al. Longitudinal patterns in the prevention of osteoporosis in glucocorticoid-treated patients. Arthritis Rheum 52: 2485-2494, 2005.

24. Mohammad A, Ryan JG, Ralph N, Ryan C, O'Connell PG. Improving trends in glucocorticoid-induced osteoporosis management: 2002 to 2006. Clin Exp Rheumatol 25: 728-733, 2007.

25. Nomura H, Nakayama T. The Japanese healthcare system: The issue is to solve the "tragedy of the commons" without making another. BMJ 331: 648-649, 2005.

26. Nakayama T. What are "clinical practice guidelines"? J Neurol 254 (Suppl 5): 2-7, 2007.

27. Sambrook PN, Kotowicz M, Nash P, et al. Prevention and treatment of glucocorticoid-induced osteoporosis: a comparison of calcitriol, vitamin D plus calcium, and alendronate plus calcium. J Bone Miner Res 18: 919-924, 2003.

28. de Nijs RN, Jacobs JW, Lems WF, et al. Alendronate or alfacalcidol in glucocorticoid-induced osteoporosis. N Engl J Med 355: 675-684, 2006.

29. Saag KG, Shane E, Boonen $S$, et al. Teriparatide or alendronate in glucocorticoid-induced osteoporosis. N Engl J Med 357: 20282039, 2007.

30. Grossman JM, Gordon R, Ranganath VK, et al. American College of Rheumatology 2010 recommendations for the prevention and treatment of glucocorticoid-induced osteoporosis. Arthritis Care Res (Hoboken) 62: 1515-1526, 2010.

31. Guzman-Clark JR, Fang MA, Sehl ME, Traylor L, Hahn TJ. Barriers in the management of glucocorticoid-induced osteoporosis. Arthritis Rheum 57: 140-146, 2007.

(C) 2011 The Japanese Society of Internal Medicine http://www.naika.or.jp/imindex.html 